\title{
Seasonal parasitism and host specificity of Trissolcus japonicus in northern China
}

\author{
Jinping Zhang ${ }^{1} \cdot$ Feng Zhang $^{1} \cdot$ Tara Gariepy $^{2} \cdot$ Peter Mason $^{3} \cdot$ Dave Gillespie $^{4}$. \\ Elijah Talamas $^{5,7} \cdot$ Tim Haye $^{1,6} \mathbb{C}_{\mathbb{C}}$
}

Received: 11 February 2017/Revised: 4 April 2017 / Accepted: 7 April 2017/Published online: 18 April 2017

(C) The Author(s) 2017. This article is an open access publication

\begin{abstract}
The brown marmorated stink bug, Halyomorpha halys (Stål), native to China, Japan, and Korea, has emerged as a harmful invasive pest of a variety of crops in North America and Europe. The Asian egg parasitoid Trissolcus japonicus has been identified as the most promising agent for classical biological control of invasive $H$. halys populations. A 4-year study evaluated the fundamental and ecological host ranges of T. japonicus as well as its phenology and impact on $H$. halys populations in fruit orchards in its native range in northern China. In laboratory no-choice tests, developmental suitability of eight nontarget host species for T. japonicus was demonstrated by the successful production of progeny on the majority ( $>85 \%$ ) of non-target host species tested. In field-collected,
\end{abstract}

Special Issue: The brown marmorated stink bug Halyomorpha halys: an emerging pest of global concern.

Communicated by M. Traugott.

Electronic supplementary material The online version of this article (doi:10.1007/s10340-017-0863-y) contains supplementary material, which is available to authorized users.

Tim Haye

t.haye@cabi.org

1 MoA-CABI Joint Laboratory for Bio-safety, 2

Yuanmingyuan West Road, Beijing 100193, China

2 London Research and Development Centre, Agriculture and Agri-Food Canada, 1391 Sandford Street, London, ON N5V 4T3, Canada

3 Ottawa Research and Development Centre, Agriculture and Agri-Food Canada, 960 Carling Avenue, Ottawa, ON K1A 0C6, Canada

4 Agassiz Research and Development Centre, Agriculture and Agri-Food Canada, 6947 Highway 7, Agassiz, BC V0M 1A0, Canada naturally laid egg masses, T. japonicus was the most abundant parasitoid associated with $H$. halys and Dolycoris baccarum, but was also sporadically found in Plautia crossota. Furthermore, it was regularly reared from sentinel egg masses of Menida violacea, Arma chinensis, and Carbula eoa. The only species that did not support development in the laboratory and field was Cappaea tibialis. Besides the benefit of having a high impact on $H$. halys populations in Northern China, the risk assessment conducted in the area of origin indicates that native Pentatomidae in North America and Europe could be negatively impacted by $T$. japonicus. Whether the benefits of $T$. japonicus outweigh the possible risks will have to be evaluated based on the outcome of additional host range studies in the two invaded regions.

Keywords Egg parasitoid · Biological control · Brown marmorated stink bug - Ecological host range .

Fundamental host range

5 Systematic Entomology Laboratory, USDA-ARS c/o NMNH, Smithsonian Institution, 10th \& Constitution Ave NW, MRC 168, Washington, DC 20560, USA

6 CABI, Rue des Grillons 1, 2800 Delemont, Switzerland

7 Present Address: Florida State Collection of Arthropods, Florida Department of Agriculture and Consumer Services, Division of Plant Industry, 1911 SW 34th St., Gainesville, FL 32608, USA 


\section{Key message}

- The Asian egg parasitoid Trissolcus japonicus is considered the most promising species for classical biological control of Halyomorpha halys

- We investigated the fundamental and ecological host range of $T$. japonicus in northern China to define its host specificity

- T. japonicus does successfully develop on Pentatomidae other than its intended target, $H$. halys, under laboratory and field conditions

- Negative impacts on native non-target species must be further studied to determine whether the benefit outweighs the risk if $T$. japonicus is released outside of Asia

- The accidental establishment of T. japonicus in North America provides an opportunity to monitor its impacts on non-target species and $H$. halys and to quantify the risks and benefits.

\section{Introduction}

The brown marmorated stink bug, Halyomorpha halys (Stål) (Hemiptera: Pentatomidae), native to subtropical and temperate areas in East Asia (China, Japan, and Korea), has emerged as a harmful invasive pest of a variety of crops in North America and Europe (Hoebeke and Carter 2003; Haye et al. 2014a, 2015b). Bioclimatic models further suggest that favourable conditions for establishment of $H$. halys exist in South America, southern Africa, and Oceania (Zhu et al. 2012; Haye et al. 2015b). The development of large pest populations outside its native range has recently generated secondary invasions within Europe and has accelerated the global spread of this pest, primarily through human-mediated activities (Gariepy et al. 2013, 2015).

In Asia, North America, and Europe, H. halys is highly polyphagous, feeding on a wide variety of native and exotic host plants, including many economically important field and tree crops, vegetables, ornamentals, herbaceous perennials, shrubs, and forest trees (Lee et al. 2013; Haye et al. 2014b; Martinson et al. 2015; Bergmann et al. 2016). Damage is caused by feeding by the adults and nymphs on fruits, buds, leaves, and stems. In the mid-Atlantic region of the USA, $H$. halys has become one of the most significant pests in apple production, causing $>\$ 37$ million in losses in 2010 (United States Apple Association 2010). In recently invaded areas in Europe and Eurasia, including northern Italy and western Georgia, severe damage has been observed in pear (Pyrus communis L.) and hazelnut (Corylus avellana L.) orchards since the arrival of $H$. halys (Bariselli et al. 2016).
Currently, chemical control is the most widely used tactic for managing $H$. halys in the Northeast of the USA; however, the most effective insecticides are generally broad spectrum in their activity, disrupting natural enemies of other pests, and thus negatively interfering with existing IPM programs (Leskey et al. 2012a, b). More environmentally friendly and self-sustaining control measures, such as conservation of existing natural enemies in invaded areas, introduction of specialized egg parasitoids from Asia for classical biological control, and the use of indigenous parasitoids for augmentative control, are currently being investigated and may become important management tools in the near future for area-wide control of $H$. halys.

In its native range, eggs of $H$. halys are attacked by a complex of species in the genera Trissolcus, Telenomus, Ooencyrtus (Platygastridae), and Anastatus (Eupelmidae), whereas nymphs and adults are rarely parasitized (Arakawa and Namura 2002; Yang et al. 2009; Lee et al. 2013). Within the egg parasitoid guild, Trissolcus japonicus (Ashmead) (syn. Trissolcus halyomorphae Yang; Talamas et al. 2013) has been identified as most promising for classical biological control of invasive $H$. halys populations, causing high levels of parasitism in China (Yang et al. 2009). Single T. japonicus females lay 42 eggs on average and prefer to oviposit in eggs no older than 1-3 days (Qiu et al. 2007). The wasp reproduces much more rapidly than its host $H$. halys, completing development from egg to adult within 10.5 and 7.3 days at 25 and $30{ }^{\circ} \mathrm{C}$, respectively. The number of degree days required for completion of development is $132.5 \mathrm{DD}$, suggesting that $T$. japonicus could potentially have up to 10 generations per year in southern China (Qiu et al. 2007; Yang et al. 2009). In addition, offspring are heavily female biased, and the parasitoids overwinter as adults, which are chill-intolerant, but appear more cold-tolerant than their hosts, H. halys (Santacruz et al. 2017). Besides these desirable attributes for potential biological control agents, Qiu (2007) reported that $T$. japonicus was also successfully reared on three other pentatomid species, Erthesina fullo (Thunberg), Dolycoris baccarum (L.), and Plautia crossota (Dallas), in the laboratory, suggesting that it may have a broad host range.

In the past decade, most countries have implemented strict regulatory requirements for the importation and release of natural enemies, with detailed risk assessment and host range studies required as part of a petition process for agent approval (Mason et al. 2013). Current practices include a comparison of field-generated host range data (ecological host range) from the native range of the biocontrol agent with that generated in laboratory studies (fundamental host range) (Van Driesche and Reardon 2004; Bigler et al. 2006; van Lenteren et al. 2006). 
Laboratory host specificity tests with North American and European Pentatomidae species are currently being conducted by USDA-ARS, CABI, and other laboratories, respectively, but the ecological host range of T. japonicus and its seasonal impact on $H$. halys populations in Asia are still poorly understood. Here, we present results from a 4-year study evaluating the fundamental and ecological host ranges of $T$. japonicus in its native range in northern China and elucidating its phenology and impact on $H$. halys populations in fruit orchards throughout the season. We use these results to predict the ecological host range and possible non-target impacts of T. japonicus in areas invaded by $H$. halys. These predictions are particularly relevant due to the recent adventive establishment of $T$. japonicus in North America (Talamas et al. 2015), as this provides the opportunity to validate the predictions of this study with the realized, 'post arrival', host range as it manifests over time. However, we cannot exclude the possibility that the $T$. japonicus strains not deliberately released to North America may differ in terms of host specificity from the Chinese strain tested here.

\section{Materials and methods}

\section{Fundamental host range}

\section{Rearing of target host species}

A colony of $H$. halys was established from adults collected in Beijing and Hebei provinces (Table 1) and maintained at the Joint Laboratory of the Ministry of Agriculture-Centre for Agriculture and Biosciences International (MoACABI), Beijing, China, in continuous rearing on a diet of green bean pods (Phaseolus vulgaris L.) and fresh ears of corn (Zea mays L.) at $25 \pm 1{ }^{\circ} \mathrm{C}, 60-70 \% \mathrm{RH}$ and $16: 8$

Table 1 Non-target species from the family Pentatomidae selected for host specificity tests with Trissolcus japonicus

\begin{tabular}{|c|c|c|c|}
\hline Test species & Host plant & Selection criteria & $\begin{array}{l}\text { Origin of laboratory } \\
\text { cultures }\end{array}$ \\
\hline \multicolumn{4}{|l|}{ Subfamily: Pentatominae } \\
\hline \multicolumn{4}{|l|}{ Tribe: Cappaeini } \\
\hline Halyomorpha halys Stål & $\begin{array}{l}\text { Robinia pseudoacacia L.; } \\
\text { Prunus persica }(\text { L.) Batch }\end{array}$ & Target & $\begin{array}{l}\text { Langfang (Hebei Province) } \\
\text { Changping, Lengquan } \\
\text { (both Beijing City) }\end{array}$ \\
\hline Cappaea tibialis Hsiao and Cheng & Robinia pseudoacacia $\mathrm{L}$. & $\begin{array}{l}\text { Habitat and host plant } \\
\text { overlap, close } \\
\text { relatedness }\end{array}$ & $\begin{array}{l}\text { Baiwang Mountain } \\
\text { (Beijing City) }\end{array}$ \\
\hline Homalogonia obtusa (Walker) & Malus baccata (L.) Borkh. & $\begin{array}{l}\text { Habitat and host plant } \\
\text { overlap, close } \\
\text { relatedness }\end{array}$ & $\begin{array}{l}\text { Miaofeng Mountain } \\
\text { (Beijing City) }\end{array}$ \\
\hline \multicolumn{4}{|l|}{ Tribe: Carpocorini } \\
\hline Dolycoris baccarum (L.) & Various weeds & $\begin{array}{l}\text { Habitat overlap, } \\
\text { literature host record }\end{array}$ & $\begin{array}{c}\text { Langfang (Hebei Province) } \\
\text { Lengquan (Beijing City) }\end{array}$ \\
\hline \multicolumn{4}{|l|}{ Tribe: Halyini } \\
\hline Erthesina fullo (Thunberg) & $\begin{array}{l}\text { Paulownia tomentosa (Thunb.) } \\
\text { Steud. }\end{array}$ & $\begin{array}{l}\text { Habitat and host plant } \\
\text { overlap, literature host } \\
\text { record }\end{array}$ & $\begin{array}{l}\text { Liuzhou } \\
\text { (Guangxi Province) }\end{array}$ \\
\hline \multicolumn{4}{|l|}{ Tribe: Eysarcorini } \\
\hline Carbula eoa (Bergroth) & Artemisia аппиа $\mathrm{L}$. & Habitat overlap & $\begin{array}{l}\text { Miaofeng Mountain } \\
\text { (Beijing City) }\end{array}$ \\
\hline \multicolumn{4}{|l|}{ Tribe: Antestiini } \\
\hline Plautia crossota Fabricius & Morus alba $\mathrm{L}$. & $\begin{array}{l}\text { Habitat and host plant } \\
\text { overlap }\end{array}$ & $\begin{array}{r}\text { Lengquan, Shujiatuo } \\
\text { (both Beijing City) }\end{array}$ \\
\hline \multicolumn{4}{|l|}{ Tribe: Menedini } \\
\hline Menida violacea Motschulsky & Morus alba $\mathrm{L}$. & $\begin{array}{l}\text { Habitat and host plant } \\
\text { overlap }\end{array}$ & $\begin{array}{l}\text { Yangtai Mountain } \\
\text { (Beijing City) }\end{array}$ \\
\hline \multicolumn{4}{|l|}{ Subfamily: Asopinae } \\
\hline \multicolumn{4}{|l|}{ Tribe: Asopini } \\
\hline Arma chinensis (Fallou) & (Predatory species) & Beneficial species & $\begin{array}{l}\text { Sino-American Biological } \\
\text { Control Laboratory } \\
\text { culture, Beijing }\end{array}$ \\
\hline
\end{tabular}


L:D in gauze cages $(60 \times 60 \times 60 \mathrm{~cm})$. In summer, freshcut branches of peach [Prunus persica (L.) Batsch] trees were offered as an additional food source and oviposition substrate and changed when needed. Leaves with newly laid eggs were collected from the adult cages daily and maintained under the same conditions in separate nymphal rearing cages.

\section{Selection, source, and rearing of non-target host species}

The selection of non-target hosts for laboratory testing was based on phylogenetic criteria, accessibility, sympatry of target and non-target species, and information on the parasitoid biology available from the literature (Table 1) (Kuhlmann et al. 2006).

Native non-target stink bugs (Hemiptera: Pentatomidae) were collected from their host plants in the spring of each year from various locations in the Beijing and Guangxi provinces (Table 1). All herbivorous species were fed with beans and corn or their associated host plants (Table 1) and kept under the same conditions as described above. Adults of the predatory species, Arma chinensis (Fallou), were obtained from the Sino-American Biological Control Laboratory in Beijing and kept in small 0.5-1 plastic containers. Pupae of the Chinese oak moth, Antheraea pernyi GuérinMéneville (Lepidoptera: Saturniidae), were removed from their surrounding cocoons and placed on the gauze lids of each container as food source. Egg masses of each non-target species were collected from rearing cages on a daily basis.

\section{Source and rearing of Trissolcus japonicus}

Trissolcus japonicus was reared from sentinel $H$. halys egg masses exposed in a fruit orchard at Lengquan, Beijing Province. Laboratory colonies were maintained in acrylic cages $(25 \times 25 \times 25 \mathrm{~cm})$ at $25 \pm 1{ }^{\circ} \mathrm{C}, 60-70 \% \mathrm{RH}$ and 16:8 L:D. Parasitoid colonies were fed daily with $10 \%$ honey water solution and provided with newly laid egg masses of $H$. halys for oviposition. Parasitized egg masses were moved to a separate cage when parasitoid adults were close to emergence, as indicated by the dark colouration of the egg masses. Newly emerged wasps were kept together for two days to ensure mating had occurred and used for testing when they were three days old. Upon the initial establishment of the laboratory colonies of $T$. japonicus, specimens were sent to the Systematic Entomology Laboratory, USDA-ARS (USA), and their identification was verified by E. Talamas.

\section{Laboratory no-choice tests}

The no-choice tests were designed to determine whether egg masses of non-target hosts were suitable for parasitoid development. In each experimental set-up, similar numbers of randomly selected, naïve, 2-3-day-old $T$. japonicus females were separately tested simultaneously on egg masses of the target $H$. halys (control) and the non-target species listed in Table 1 (between 4 and 61 replicates per non-target species). All egg masses and parasitoids were taken from the laboratory colonies described above. Each parasitoid female was offered a single, newly laid $(0-48 \mathrm{~h})$ egg mass of either the target or non-target species. Egg masses were exposed to single females for $24 \mathrm{~h}$ in a Petridish experiment ( $5 \mathrm{~cm}$ diameter). A cotton wick saturated in honey water was provided as a food source for the wasps. After $24 \mathrm{~h}$, the wasps were removed and the eggs were reared at $25 \pm 1{ }^{\circ} \mathrm{C}, 60-70 \% \mathrm{RH}$ and 16:8 L:D light until all parasitoids and/or nymphs had emerged, up to 3 weeks. The number of emerged parasitoids, nymphs and dead eggs (='no emergence') was recorded as well as the sex ratio of the parasitoid offspring.

\section{Ecological host range}

\section{Field-collected egg masses}

In northern China, surveys for stink bug egg masses were conducted at 12 sites in Beijing and Hebei provinces from 2012 to 2015 to document the egg parasitoid assemblages of $H$. halys and co-occurring non-target Pentatomidae.

Sites included agricultural research stations with a wide variety of trees and shrubs (Langfang, Beijing), fruit orchards with a mix of peach, cherry (Prunus avium L.), mulberry (Morus alba L. and M. nigra L.), and apple (Malus pumilla Miller) trees (Haidian, Hengshui, Lengquan, Shengshuyuan, Shujiatuo), or natural forests and parks (Baiwang Mountain, Miaofeng Mountain, Fragrant Hills Park, Xishanlinyu, Xiaojiahe). Sites with high densities of $H$. halys adults (e.g. Langfang) were visited more than once per year. At each site, the underside of leaves of trees and shrubs were carefully inspected for the presence of stink bugs and their egg masses. Leaves with egg masses were removed from their host plants and taken back to the MoA-CABI Joint Laboratory in Beijing, where they were maintained individually in small Petri dishes $(5 \mathrm{~cm}$ diameter) at $25 \pm 1{ }^{\circ} \mathrm{C}, 60-70 \% \mathrm{RH}$ and 16:8 L:D until all parasitoids and/or nymphs had emerged, up to 7 weeks. Emerged parasitoids were transferred into ethanol, and subsamples were sent to E. Talamas (Systematic Entomology Laboratory, USDA-ARS, USA) and M. C. Bon (European Biological Control Laboratory, Montpellier, France) for morphological and molecular identification, respectively. Egg masses of non-target species were identified by comparing field-collected egg masses with egg masses from our laboratory colonies of non-target species. If it was not possible to identify an egg mass based on 
morphological comparison with our laboratory colonies, hatched nymphs were reared to the adult stage for identification. Taxonomic guidance was provided by $\mathrm{W}$. Bu (Nankai University, China).

\section{Exposure of sentinel egg masses}

As egg masses of Pentatomidae are often well-camouflaged and difficult to locate in the foliage of trees and shrubs, it can be challenging to find sufficient numbers in the field. To supplement the information provided by naturally laid, field-collected egg masses, sentinel egg masses from laboratory-reared non-target species were exposed at various sites to determine whether non-target egg masses would be attacked by $T$. japonicus under field conditions.

In 2013, sentinel eggs of $H$. halys, D. baccarum, and $P$. crossota were exposed weekly in two orchards near Lengquan (N40 $02^{\prime} 06^{\prime \prime}$; E116 $12^{\prime} 41^{\prime \prime}$ ) and Shengshuyuan $\left(\mathrm{N} 40^{\circ} 03^{\prime} 07^{\prime \prime}\right.$; E116 $\left.06^{\prime} 44^{\prime \prime}\right)$, both located in the suburbs of Beijing City. Halyomorpha halys eggs served as a control for the presence of T. japonicus at the selected sites. At the mixed orchard near Lengquan, peach leaves with newly laid egg masses (collected the same day or stored at $10{ }^{\circ} \mathrm{C}$ for maximum $48 \mathrm{~h}$ to prevent development) were stapled on the underside of leaves of randomly selected peach, white mulberry (M. alba) or jujube trees (Ziziphus jujube Mill.) in the interior of the orchard. At Shengshuyuan, egg masses were exclusively exposed on mulberry trees. The number of egg masses exposed per week and per species varied with availability, ranging from 4 to 10 . Egg exposure at Lengquan and Shengshuyuan started in early May and early June, respectively, and lasted until early September.

In 2014, six sites located in the suburbs of Beijing City were selected for sentinel egg exposure, including three orchards [Lengquan (peach/cherry/mulberry orchard), Sujiatuo (peach/cherry orchard; N40 $03^{\prime} 58^{\prime \prime}$; E116 $06^{\prime} 46^{\prime \prime}$ ), Yantai mountain (peach orchard; $\mathrm{N} 40^{\circ} 04^{\prime} 15.84^{\prime \prime}$; E116 $\left.04^{\prime} 53.12\right)$ ], and three natural forest sites in the western mountains near Beijing [Beianhe village (N4004' 8.5.52"; E116 $\left.04^{\prime} 54.43^{\prime \prime}\right)$, Baiwang Mountain $\left(\mathrm{N} 40^{\circ} 01^{\prime} 53^{\prime \prime}\right.$; E $\left.116^{\circ} 15^{\prime} 32^{\prime \prime}\right)$, Fragrant Hills $\left(\mathrm{N} 40^{\circ} 03^{\prime} 58.51^{\prime \prime}\right.$; E116 $\left.\left.03^{\prime} 0.18^{\prime \prime}\right)\right]$. Egg masses of H. halys, D. baccarum, P. crossota, Menida violacea Motschulsky, and $A$. chinensis were exposed every two weeks between May and August at each of the selected sites. At Fragrant Hills, egg masses were exposed on smoke trees [Cotinus coggygria (Scopoli)], whereas at all other sites, egg masses were stapled to peach trees.

Exposed egg masses were marked with coloured flagging tape and retrieved after five days. Egg masses were kept separately in small Petri dishes at $25 \pm 1{ }^{\circ} \mathrm{C}, 60-70 \%$ RH and 16:8 L:D until all parasitoids and/or nymphs had emerged.

\section{Statistical analysis}

In small arena no-choice tests, the proportion of females successfully producing offspring (=suitability) and the proportion of male and female offspring (sex ratio) were
Fig. 1 Percentage of $T$. japonicus females successfully parasitizing egg masses of Halyomorpha halys (white bars) and non-target hosts (black bars) in small arena no-choice tests. The number of tested females is given in brackets for each species. Bars marked with asterisks indicate a significant difference between groups (Pearson Chi-Square test: $*_{p}<0.05 ; * * p<0.01$; $* * * p<0.001 ; n s$ not significantly different). Due to the low number of replicates, $E$. fullo was not included in the analysis (-)

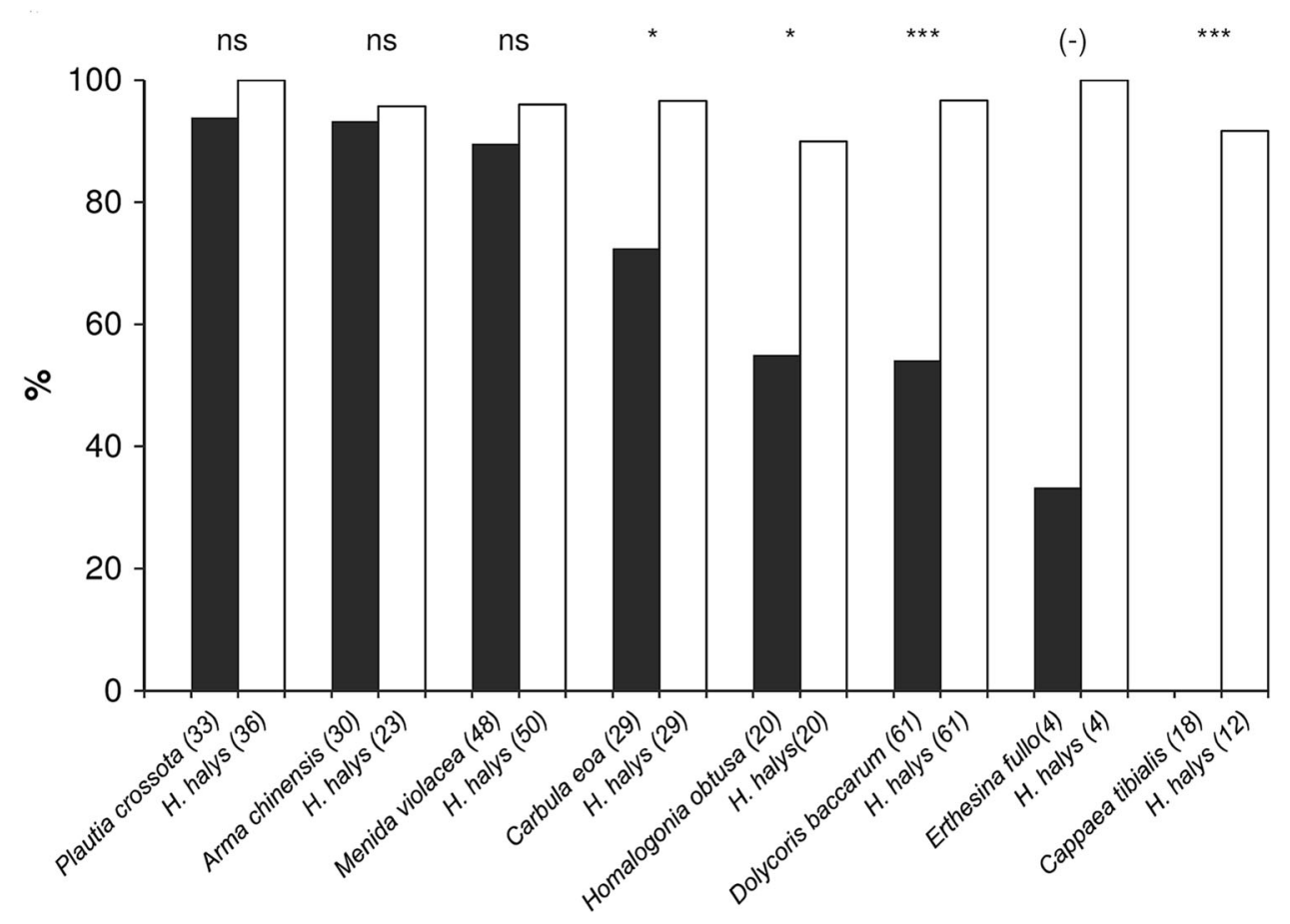


compared among treatments (=host species) using a Pearson Chi-Square test. To compare parasitism levels, only egg masses from which parasitoids had emerged were included in the analysis. Parasitism levels (=proportion of eggs producing offspring within an egg mass) were calculated by the number of emerged parasitoids divided by the total number of eggs in the mass. Egg mortality was defined as the proportion of eggs from which neither nymphs nor parasitoids had emerged. The average successful development and the average egg mortality in the control (H. halys) and the different treatments (non-target hosts) were compared pairwise, using Mann-Whitney U tests. All statistical analyses were carried out with the SPSS $^{\circledR} 20.0$ software package (IBM Corp. 2013).

\section{Results}

\section{Fundamental host range}

In total, seven out of eight non-target hosts were accepted and suitable for parasitoid development (Fig. 1). Levels of suitability (=proportion of females successfully producing offspring from $>50 \%$ of eggs in an egg mass) were not significantly different for three non-target species, $P$. crossota, $M$. violacea and A. chinensis, than for the target host $H$. halys. Suitability of the target $H$. halys (controls) was generally high, varying between 90 and 100\% (average
95.8\%). In contrast to the $H$. halys controls, rates of suitability of Carbula eoa (Bergroth) (72.4\%) (Pearson ChiSquare test, $\left.\chi^{2}=6.444, p=0.011\right)$, Homologonia obtusa (Walker) $(55.0 \%)\left(\chi^{2}=6.144, p=0.013\right)$, and D. baccarum $(54.1 \%)\left(\chi^{2}=29.881, p<0.0001\right)$ were significantly lower. The only species from which no $T$. japonicus offspring developed was the closely related $C$. tibialis, which belongs to the same tribe (Cappaeini) as $H$. halys. For E. fullo, test data are preliminary as only four females could be tested, and only one successfully produced offspring.

Parasitism levels (=proportion of eggs producing offspring within an egg mass) were generally high $(>70 \%)$ for all species tested (Fig. 2), but not significantly different between $H$. halys (93.8\%) and the two non-target hosts $M$. violacea $(89.9 \%)$ and C. eoa (96.8\%) (Mann-Whitney tests, $Z=-1.192 ; Z=-1.343$, both $p>0.05$ ). In contrast, in P. crossota $(Z=-2.370, p=0.018)$, A. chinensis $(Z=-3.569, \quad p \leq 0.001), \quad H . \quad$ obtusa $\quad(Z=-2.862$, $p=0.004)$, and $D$. baccarum $(Z=-4.635, p \leq 0.001)$, parasitism levels were significantly lower than in the $H$. halys controls, and egg mortality (=no emergence of nymphs or parasitoids) for these four species was significantly higher (Fig. 2).

The proportion of $T$. japonicus females emerging from H. halys eggs (control) was $70.6 \%$, whereas for C. eoa $\left(55.1 \%, \chi^{2}=23.989, p<0.0001\right)$ and H. obtusa $(40.8 \%$, $\left.\chi^{2}=47.534, \quad p<0.0001\right)$, it was significantly lower
Fig. 2 Mean $( \pm \mathrm{SE})$ proportion of successfully parasitized eggs (=parasitoid emergence) and egg mortality (no emergence of nymphs or parasitoids) within individual egg masses in small arena no-choice tests. The number of replicates is given in brackets for each species. Bars marked with asterisks indicate a significant difference between control (H. halys) and treatments (non-target species) groups, (Mann-Whitney test: $p<0.05 ; * * p<0.01$; $* * * p<0.001 ; n s$ not significantly different)

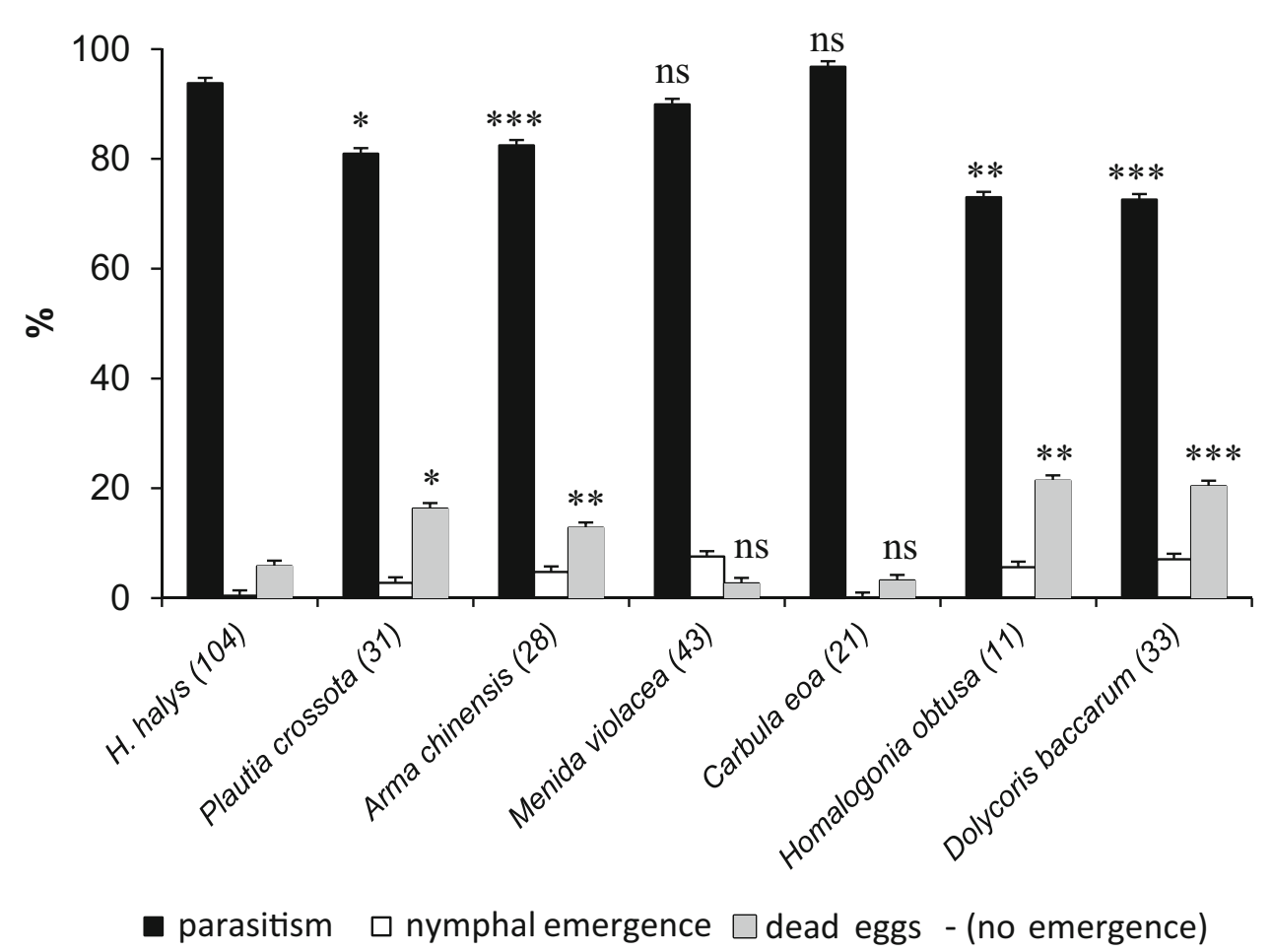


Fig. 3 Sex ratio of offspring reared from $H$. halys and six no. of individuals emerged); bars marked with asterisks indicate a significant difference treatments (non-target species) test: $p<0.05 ; * * p<0.01$; $* * * p<0.001 ; n s$ not significantly different) non-target hosts (in brackets: between control (H. halys) and groups (Pearson Chi-Square

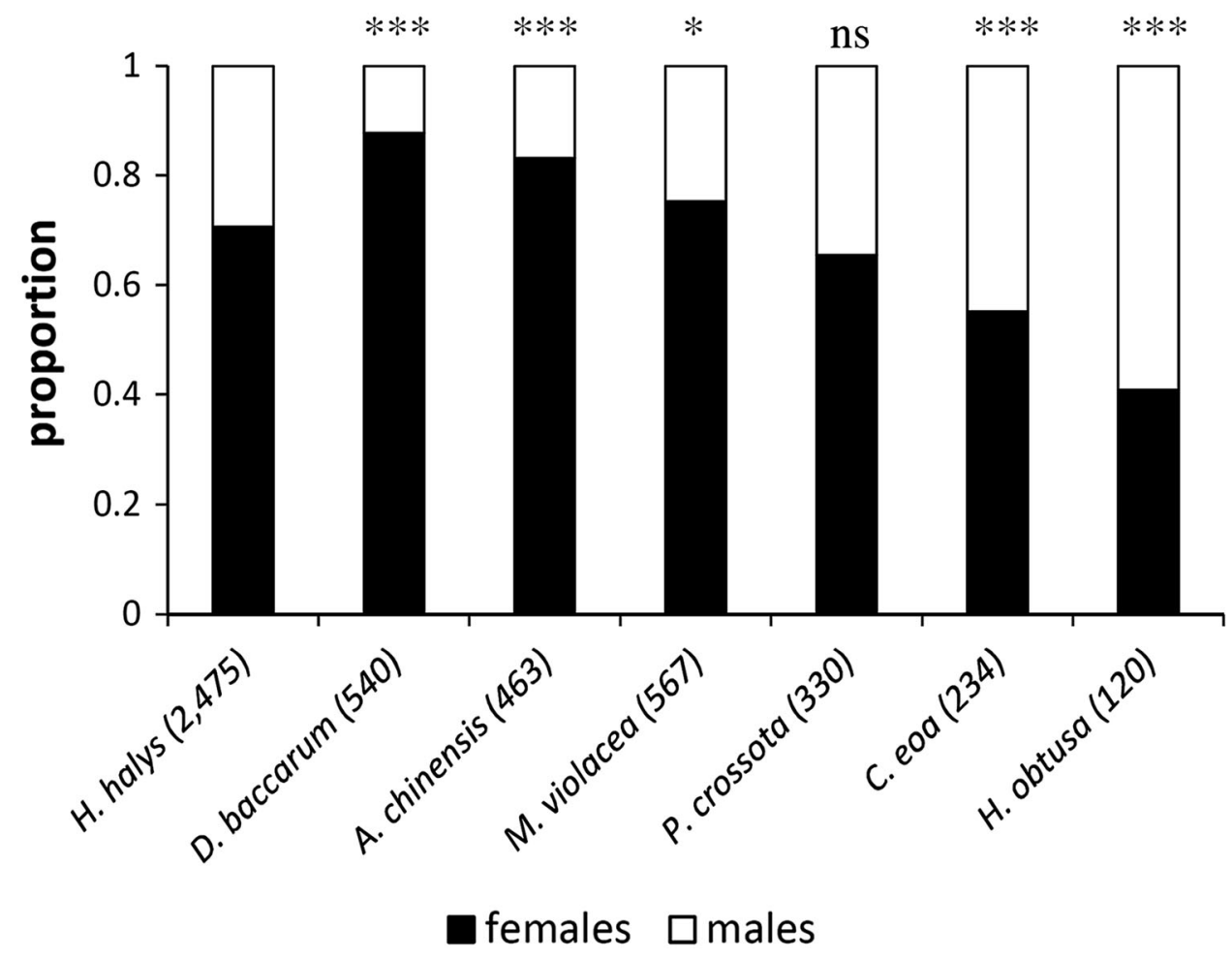

Fig. 4 Species composition of parasitoids reared from fieldcollected egg masses of Halymorpha halys and three non-target species (in brackets: no. of emerged parasitoids). For details, see supplementary Table 1

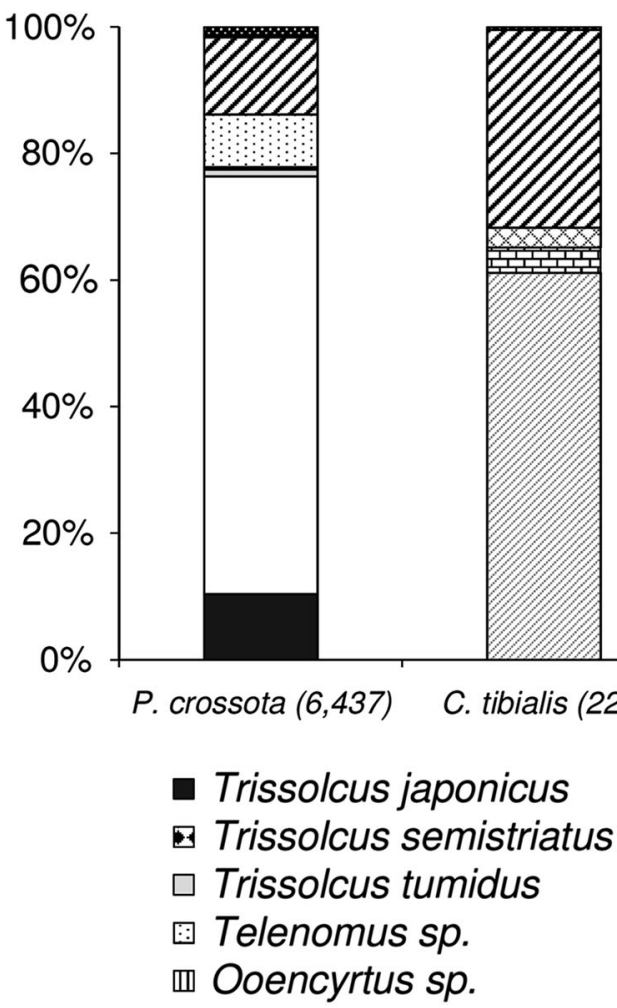

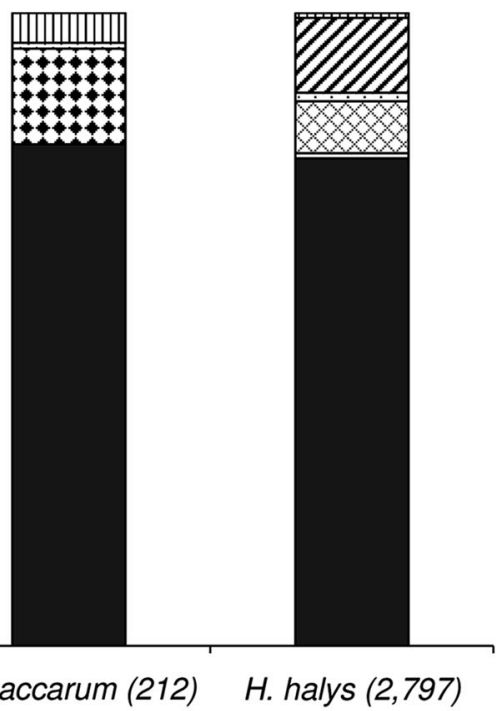

- Trissolcus plautiae

- Trissolcus sp.

Anastatus sp.

Acroclisoides sp.
Trissolcus cultratus
(Fig. 3). From eggs of M. violacea $\left(75.3 \%, \chi^{2}=5.046\right.$, $p=0.025)$, A. chinenis $\left(83.1 \%, \chi^{2}=30.944, p<0.0001\right)$, and D. baccarum $\left(87.8 \%, \chi^{2}=67.536, p<0.0001\right)$, significantly more females emerged. The proportion of females emerging from $P$. crossota eggs was not significantly different from the control $\left(65.5 \%, \chi^{2}=3.650\right.$, $p=0.056)$. 


\section{Ecological host range}

\section{Field-collected egg masses}

In total, 186 out of $236 \mathrm{H}$. halys naturally laid egg masses (=5944 eggs) were parasitized $(78.8 \%)$ (Supplementary Table 1). From those egg masses, 2799 parasitoids were reared. Consistent with previous studies (Qiu et al. 2007; Yang et al. 2009), our field collections confirmed that $T$. japonicus is the most abundant egg parasitoid of $H$. halys in northern China (proportion: $77.2 \%, N=2799$ ), followed by Anastatus sp. (11.8\%) and Trissolcus cultratus (8.1\%) (Fig. 4; Supplementary Table 1). Other species such as Trissolcus plautiae (0.8\%), Telenomus sp. (1.3\%), and Ooencyrtus sp. $(0.8 \%)$ were reared sporadically. Parasitized egg masses were found on 11 different host plants, but most egg masses (46.5\%) were collected from black locust (Robinia pseudoacacia L.).

The diversity and abundance of non-target Pentatomidae in the Beijing suburbs was generally low, and thus, collecting sufficient numbers of egg masses was challenging. In total, 995 egg masses belonging to four non-target species were collected, from which a total of 6879 parasitoids were reared (supplementary Table 1). The only abundant species in fruit orchards was $P$. crossota, a common species on mulberry trees. In total, parasitoids emerged from $77.9 \%$ of the $P$. crossota egg masses $(n=891)$. The dominant egg parasitoids of $P$. crossota were $T$. plautiae $(66.0 \%)$, followed by Anastatus sp. (12.2\%) and T. japonicus (10.4\%) (Fig. 4; Supplementary Table 1). At Lengquan, where eggs of $P$. crossota were collected continuously in 2013, the proportion of $T$. japonicus increased from $0.8 \%$ in May $(n=1223)$ to $31.6 \%$ in July ( $n=316$ ), the end of the egg-laying period of $P$. crossota (Supplementary Table 1). Furthermore, $T$. japonicus comprised $79.3 \%$ of the egg parasitoid species of $D$. baccarum $(n=212)$, whereas the proportion of $T$. semistriatus was $15.1 \%$ (Fig. 4). Egg masses of $C$. tibialis $(n=71)$ were only found on black locust trees at Baiwang Mountain. There were no T. japonicus among the parasitoids emerging from eggs of $C$. tibialis $(n=1108)$, which were primarily parasitized by an undescribed Trissolcus sp. (61.2\%) and Anastatus sp. (31.3\%) (Fig. 4). Trissolcus tumidus and the rare hyperparasitoid Acroclisoides sp. (Hymenoptera: Pteromalidae) were only sporadically reared. Overall parasitism of $C$. tibialis was $20.2 \%$. Only two egg masses of $A$. chinensis were found, from which six Anastatus sp. were reared.

\section{Exposure of sentinel egg masses}

In 2013, 289, 250, and $115 \mathrm{H}$. halys egg masses were exposed on peach, mulberry, and jujube trees, respectively, in an orchard near Lengquan. In total, 13,868 eggs were recollected, from which 6767 parasitoids were reared. Trissolcus japonicus was by far the most abundant species from host eggs on all three types of trees, whereas the other parasitoid species were of minor importance. Parasitism by T. japonicus on peach trees increased from $9.2 \%$ in May to 68\% in August (Fig. 5c). On mulberry trees, parasitism was higher from the beginning (36.3\%), but peak parasitism by $T$. japonicus was also observed in August (80.1\%). Remarkably, parasitism by $T$. plautiae was more than $20 \%$ in May and June. On jujube trees, parasitism by T. japonicus was 58 and $78 \%$ in July and August, respectively, and T. japonicus still emerged in small numbers from eggs exposed in early September. Simultaneously exposed egg masses of $P$. crossota on mulberry trees were heavily parasitized by $T$. plautiae in the months May and June, whereas eggs exposed on peach trees were far less parasitized (Fig. 5b). In these months, parasitism by $T$. japonicus was low and did not exceed 10\%. However, when $T$. plautiae stopped being active in the field in July, exposed $P$. crossota eggs were exclusively parasitized by $T$. japonicus. Egg masses of D. baccarum were regularly attacked in the field and the highest parasitism levels were observed in August, ranging from 63 to 88\% (Fig. 5a). Remarkably, nearly all parasitoids reared from $D$. baccarum were T. japonicus.

At Shengshuyuan, where H. halys eggs $(n=8569)$ were only exposed on mulberry trees, $T$. japonicus was by far the most abundant species $(81.2 \%)$, followed by $T$. cultratus (16.4\%) (Fig. 6a). Parasitism levels by T. japonicus were continuously high throughout the summer, ranging from 64 to $90 \%$. Similar to the Lengquan exposure site, $P$. crossota eggs were mainly parasitized by $T$. plautiae, but parasitism by $T$. japonicus was higher in May (42 vs. $20 \%$ ) but lower in June (30 vs. $75 \%$ ). In July and August, exposed eggs of $P$. crossota and $D$. baccarum were exclusively parasitized by $T$. japonicus (Fig. 6a).

In 2014, sentinel egg exposure at Lengquan was continued, but $H$. halys egg masses were only exposed on peach trees (Fig. 6b; Supplementary Table 2). Similar to the previous year, parasitism of $H$. halys was lowest in May (22.5\%) and steadily increased until August (59.6\%). In the first three months, parasitism was exclusively caused by $T$. japonicus, confirming its importance for the natural control of $H$. halys. However, it was also the dominant parasitoid of $M$. violacea, causing 41 and $15 \%$ parasitism in June and July, respectively. Egg masses of the predatory A. chinensis were only exposed in June, but parasitism by $T$. japonicus reached $31 \%$. Overall parasitism of sentinel $D$. baccarum eggs was generally lower, and the contribution of $T$. japonicus in June and July was 9 and 1\%, respectively. Similar to 2013, sentinel eggs of $P$. crossota exposed on mulberry trees in May 2014 were primarily parasitized by 
Fig. 5 Parasitism of

a Dolycoris baccarum,

b Plautia. crossota, and

c Halyomorpha halys egg

masses exposed on three

different types of trees ( $P$ peach;

$M$ mulberry; $J$ jujube tree) at

Lengquan in 2013. Numbers in

brackets indicate the number of

egg masses exposed/recollected.

Exposure on jujube trees was

not started before July
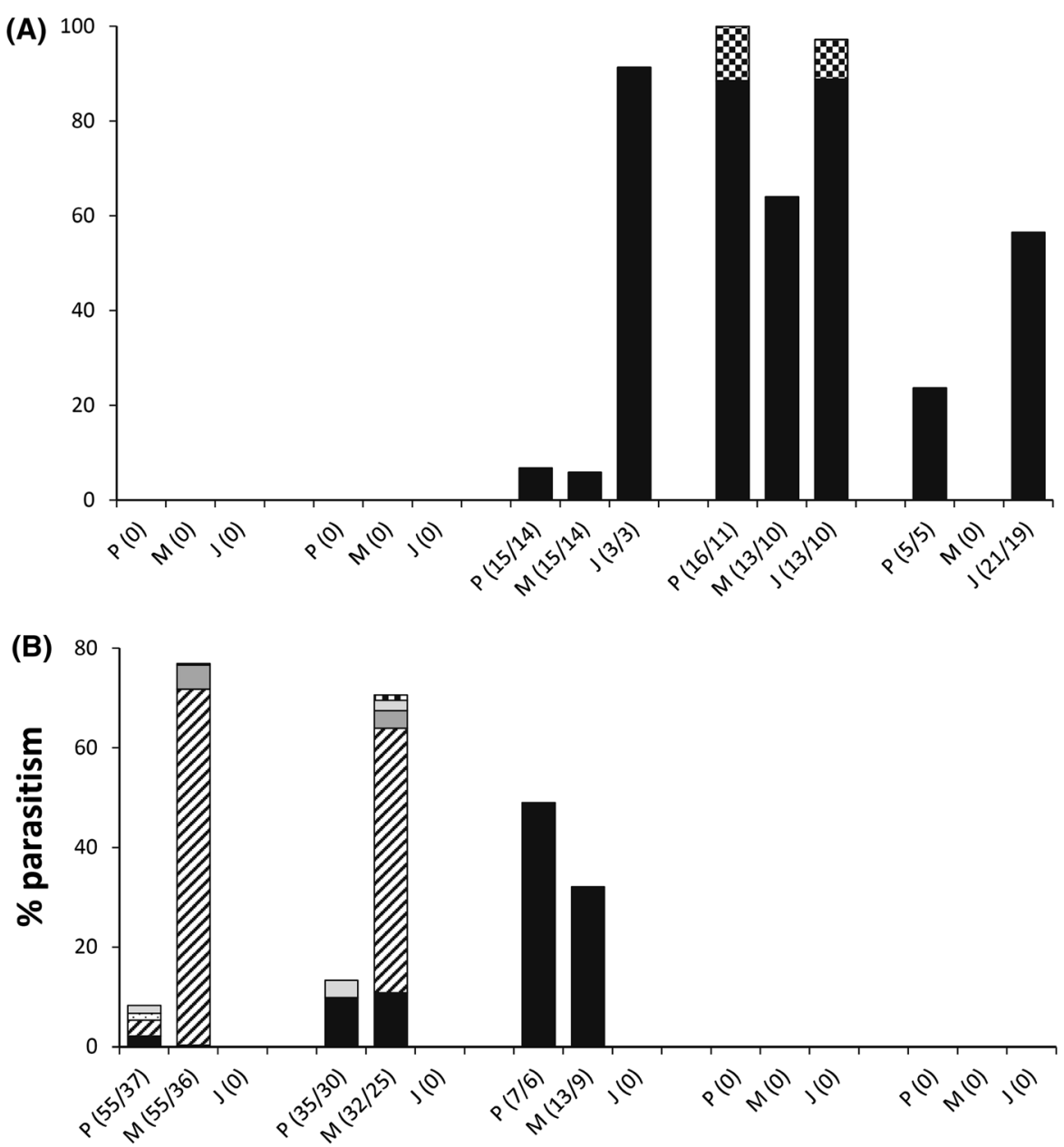

(C)

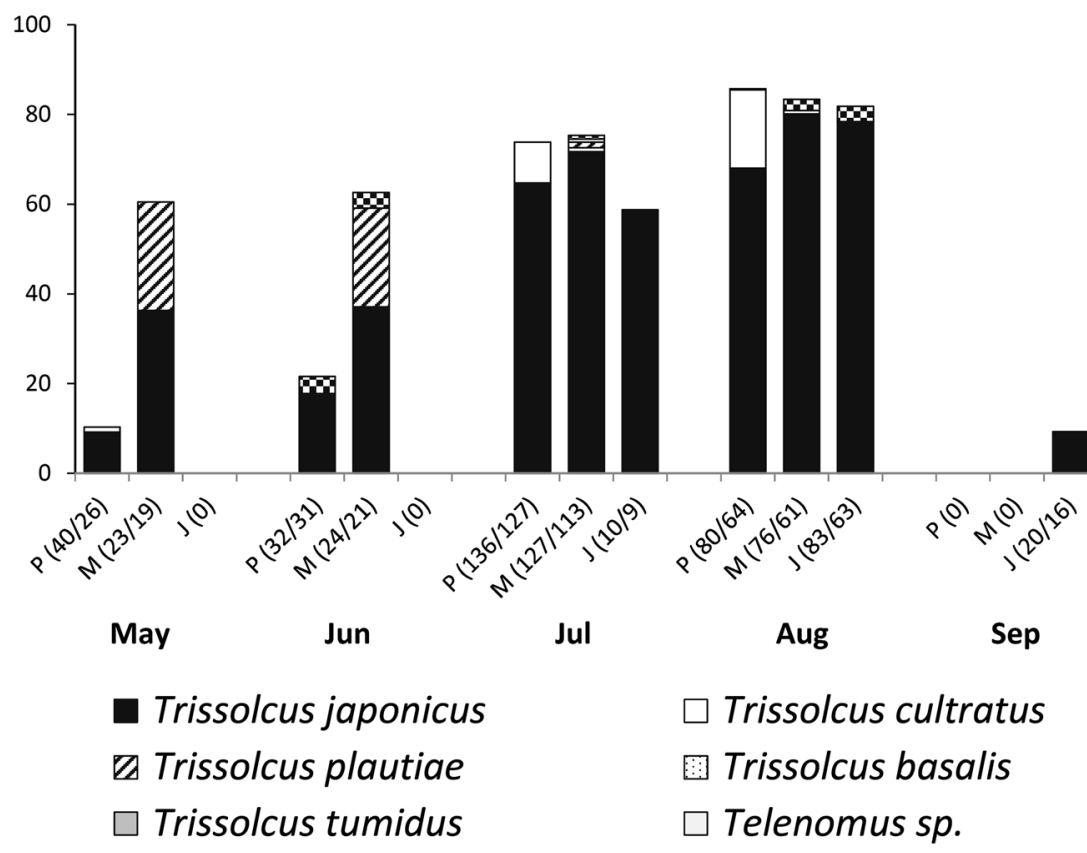

Anastatus sp. 
Fig. 6 a Parasitism of $H$. halys, $P$. fimbriata, and D. baccarum egg masses exposed on mulberry trees at Shengshuyuan in 2013 and b parasitism of Pentatomidae egg masses exposed at Lengquan in 2014. Egg masses were exposed on peach trees, except Plautia crossota which were exposed on mulberry trees. Numbers in brackets indicate the number of egg masses exposed/recollected

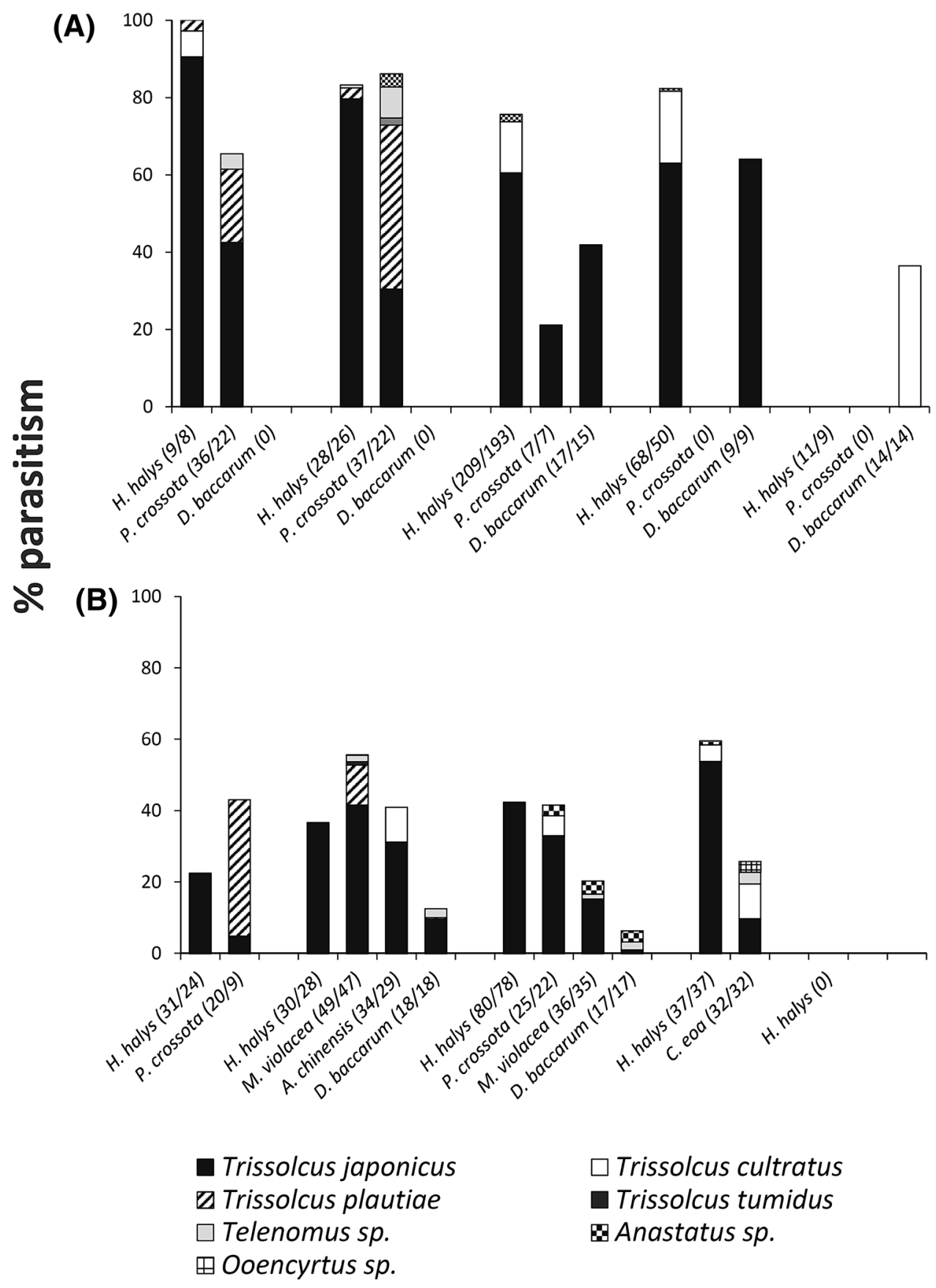

T. plautiae and the contribution of $T$. japonicus to overall parasitism was only $5 \%$. However, when $T$. plautiae was no longer present, in July, T. japonicus caused $33 \%$ parasitism.

Besides Lengquan, sentinel eggs were exposed at 5 additional sites, but the available number of eggs per month and site was often too low to give conclusive results for each site and species. As such, the data from all sites were pooled in order to provide a comprehensive overview of species composition information (Fig. 7; supplementary Table 2). In total, 1680 parasitoids were reared from sentinel $H$. halys eggs and $T$. japonicus comprised $90.1 \%$ of the parasitoid species composition. In addition, T. japonicus dominated the parasitoid complex of $M$. violacea and D. baccarum, constituting $>74$ and $>63 \%$ of the associated parasitoids, respectively. The egg parasitoid complex of $C$. eoa was dominated by Ooencyrtus sp. (36.4\%), whereas $T$. japonicus and T. cultratus were equally abundant (25.9\%). 
Fig. 7 Species composition of parasitoids reared from sentinel egg masses of Halymorpha halys and three non-target species exposed at six different sites in 2014 (in brackets: no. of emerged parasitoids). For details, see supplementary Table 2

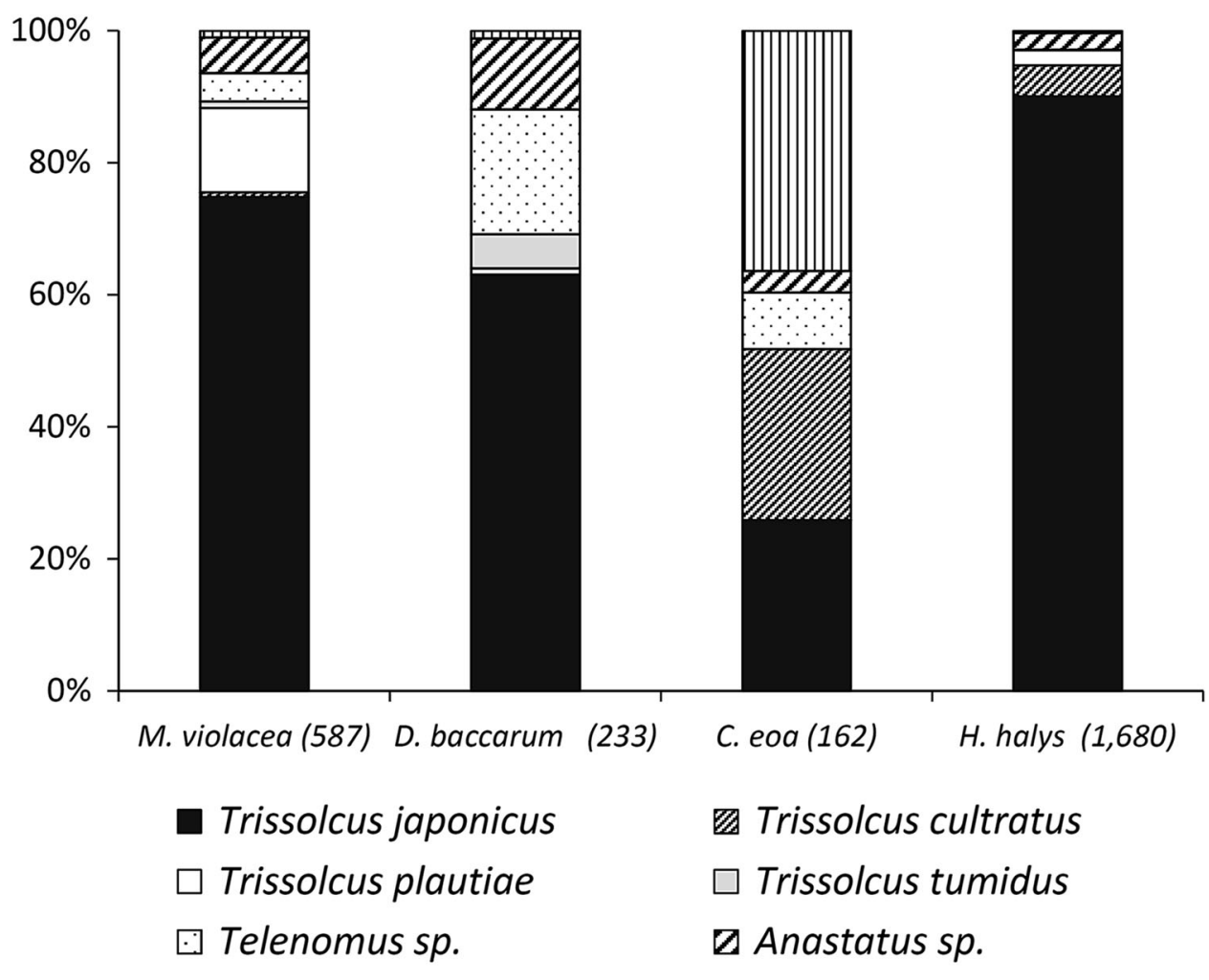

m Ooencyrtus $s p$.

\section{Discussion}

The results of our assessments of the fundamental and ecological host range of $T$. japonicus in Northern China demonstrate that $T$. japonicus does successfully develop on Pentatomidae other than the intended target for classical biological control, $H$. halys, under laboratory and field conditions. On the other hand, the present study confirmed findings of previous studies (Yang et al. 2009), demonstrating the outstanding impact of T. japonicus on $H$. halys populations with egg parasitism rates of $50-80 \%$ in the months July and August.

In laboratory no-choice tests, developmental suitability of non-target host species for $T$. japonicus was demonstrated by the successful production of progeny on seven out of eight tested non-target host species. Despite the phylogenetic diversity represented in the non-target test list (seven tribes belonging to two subfamilies), T. japonicus was able to successfully exploit representatives from each of these tribes. In fact, the suitability for parasitoid development of A. chinensis, a predatory stinkbug in the subfamily Asopinae, was not significantly different than that of the target host, $H$. halys, suggesting that $T$. japonicus may have a very broad host range within the family Pentatomidae. Interestingly, closely related species within the same tribe were either not suitable (e.g. C. tibialis) or significantly less suitable than $H$. halys (e.g. H. obtusa) in terms of the successful production of offspring. This suggests that factors other than phylogenetic relatedness influence the suitability of a host egg for T. japonicus. Furthermore, in addition to host acceptance by a parasitoid, behavioural tests are equally important as suitability tests when assessing host specificity (Duan and Messing 1997), and as such, additional studies are recommended to determine whether there are potential behavioural barriers to parasitism that may exist and preclude excessive parasitism in the field.

Although laboratory host range can serve as a predictor of non-target parasitism in the field (Barratt et al. 1997), no-choice laboratory conditions often overestimate the likely host range of a biological control agent (Haye et al. 2005). As such, the ecological host range of T. japonicus was evaluated to determine the occurrence and level of parasitism of non-target hosts in field conditions. Between 2012 and 2015, a total of 1231 naturally laid, field-collected egg masses belonging to five different pentatomid species were collected and yielded ten different parasitoid species. Trissolcus japonicus was the most abundant parasitoid associated with two of these pentatomid species, $H$. halys and $D$. baccarum, but was also sporadically found in P. crossota ( $\sim 10 \%$ of the parasitoid species composition). This is consistent with the fundamental host range results, 
which demonstrated that these hosts are physiologically suitable for development and result in a high level of parasitoid emergence $(>70 \%)$. Also consistent with the results of the fundamental host range test, $T$. japonicus was not reared from C. tibialis egg masses collected in the field, which supports the laboratory no-choice test which showed that this host is not suitable (either physiologically or behaviourally) for development.

Naturally laid, field-collected egg masses certainly provide the most reliable insights into the host selection behaviour of $T$. japonicus in field conditions, but the value of those data is often limited by the sample size. In the present study, the exposure of sentinel target and non-target egg masses at different sites during two field seasons was used as an alternative method to complement the ecological host range data. Similar to field-collected egg masses, $T$. japonicus was the dominant parasitoid associated with sentinel egg masses of $H$. halys, D. baccarum, and $M$. violacea (not represented in the field-collected egg masses). Although $C$. eoa sentinel egg masses yielded $T$. japonicus (26\% of the parasitoid species composition), it was not the dominant parasitoid species in this host. Interestingly, the proportion of $T$. japonicus from $H$. halys eggs was $>90 \%$, whereas from the non-targets, it was between 26 and $75 \%$, suggesting that these non-target hosts are less preferred. However, egg mortality was also fairly high (23-54\%) and thus the presence of $T$. japonicus may be underestimated due to host mortality making it difficult to determine whether the lower prevalence of $T$. japonicus in non-target hosts was due to reduced levels of attack or reduced suitability (resulting in host and parasitoid mortality). The fundamental host range experiments demonstrated that emergence of $T$. japonicus from $M$. violacea and $C$. eoa eggs was not significantly different than from those of $H$. halys. But, in the case of $C$. eoa, the proportion of $T$. japonicus females successfully producing progeny (i.e. indicating developmental suitability) was slightly less than those from $H$. halys. Additional laboratory studies addressing attack and suitability as well as fitness of $T$. japonicus on different non-target hosts may help fill in these gaps. Incorporating molecular tools to detect parasitoid DNA within the unhatched egg may also shed some light on the source of host mortality in field-collected egg masses (Gariepy et al. 2014).

It is important to note that the non-target sentinels were exposed in habitats and on host plants where $H$. halys naturally occurs in high numbers (e.g. peach orchards), which may increase the frequency and likelihood of encounters with $T$. japonicus. The cues used by $T$. japonicus to locate their hosts are still poorly understood. Colazza et al. (2009) demonstrated that in a similar system, Trissolcus basalis (Wollaston) perceived chemical footprints left behind by females of its host, Nezara viridula
(L.), on the plant surface as contact kairomones. If $T$. japonicus uses a similar host finding mechanism, and if chemical foot prints of naturally occurring $H$. halys females were already present on trees prior to the exposure of sentinel eggs, then this may explain the increased incidence and dominance of $T$. japonicus in the parasitoid species composition in sentinel egg masses versus naturally laid, field-collected non-target egg masses. Future non-target studies should therefore focus on the chemical ecology of $T$. japonicus, particularly on the attractiveness of chemical traces of non-target versus target species.

Remarkably, in naturally laid, field-collected egg masses each pentatomid host species had a distinct, dominant parasitoid species: $P$. crossota was predominantly attacked by $T$. plautiae; C. tibialis was predominantly attacked by $T$. tumidus; $H$. halys and D. baccarum were predominatly attacked by $T$. japonicus. This suggests the potential for resource partitioning in Trissolcus species to minimize inhost competition. This is supported by the observation of seasonal changes in the parasitoid species composition in $P$. crossota sentinel egg masses, wherein $T$. plautiae is the dominant parasitoid species in May and June, and $T$. japonicus only appears as the dominant parasitoid in this host in July, when $T$. plautiae is no longer present (Figs. 5b, 6a, b). However, this was not observed in the other non-target hosts, where sentinel eggs masses were predominantly attacked by T. japonicus. As previously mentioned, the dominance of $T$. japonicus in these nontargets may be due to the fact that they were exposed in habitats and on host plants where high densities of $H$. halys can be found and therefore may provide an overestimation of the risk to these non-target hosts. Additional studies exposing sentinel egg masses of both the target and nontarget hosts in habitats/host plants where $H$. halys is not common would provide additional insight regarding attack levels and parasitoid species composition.

Although there is clear evidence that $T$. japonicus can attack and develop in a variety of non-target Pentatomidae, the population-level impacts remain unclear. However, the concern regarding the impact of $T$. japonicus on rare and beneficial pentatomid species is valid. Populations of the endemic Hawaiian pentatomid genera Coleotichus and Oechalia declined significantly following the introduction of Trissolcus basalis for biological control of the invasive southern green stink bug, Nezara viridula (Howarth 1991). Laboratory studies on native species of concern should help predict the impact of $T$. japonicus on sensitive, rare, and beneficial species in the area(s) of proposed introduction. Our research suggests that there may be some level of habitat and/or resource partitioning in the field that results in lower levels of parasitism on non-target species versus the target species, H. halys. Gariepy and Roitberg (2013) discussed the potential of using mathematical models to 
assess the potential non-target risks associated with biological control agents. Additional studies using mathematical models that incorporate the data from fundamental and ecological host range studies may be useful to predict the outcome of non-target parasitism and provide a more detailed assessment of population-level impacts of $T$. japonicus in the area of origin and in the areas of proposed introduction.

Non-target impacts may also include those affecting parasitoid species native to the area of introduction. Abram et al. (2014) discovered that egg masses of $H$. halys were attacked by Telenomus podisi Ashmead (Hymenoptera: Scelionidae) an egg parasitoid of Podisus maculiventris (Say) (Hemiptera: Pentatomidae). However, T. podisi adult emergence from $H$. halys eggs was nil compared to $>98 \%$ for its natural host. Thus, H. halys was proposed to be a potential 'evolutionary sink' for egg parasitoids of native North American pentatomids, suggesting that parasitoid populations of native pentatomids could decline. Similarly, a recent study discovered that European strains of Trissolcus cultratus (Mayr) could not successfully develop on fresh $H$. halys eggs, whereas Chinese strains could (Haye et al. 2015a). However, it was later demonstrated that the European T. cultratus strain could emerge from $H$. halys eggs parasitized by $T$. japonicus when the latter species reaches the mature larval stage (Konopka et al. 2017), potentially reducing the impact of the evolutionary trap posed by $H$. halys.

As discussed by Duan and Messing (1997), a better understanding of the predictability of such ecological interactions can only be accomplished by increasing our knowledge of the behavioural ecology and population dynamics of the exotic biological control agent in question. The results of this study provide a piece of this puzzle and have important implications for the impact of T. japonicus in North America. Assessment of host range and attack rates in the area of origin indicates that $T$. japonicus may negatively impact a number of the native North American Pentatomidae species ( $>220$ spp. in North America Froeschner 1988). However, the natural enemy 'sink' for native parasitoid species proposed by Abram et al. (2014) could have a positive effect on populations of native pentatomids through partial 'enemy release'.

The discovery that $T$. japonicus has established adventively in the eastern and western USA (Talamas et al. 2015; Milnes et al. 2016) provides an opportunity to not only track its establishment and spread but also to assess impact on non-target Pentatomidae. Retrospective case studies on exotic biological control agents can provide information regarding the environmental effects of an agent and can be used to refine tests and protocols for predicting host specificity (Follet et al. 2000). In the case of T. japonicus, monitoring should focus on natural and urban park-type habitats where a diversity of host plants, Pentatomidae and parasitoid species, occurs (see Talamas et al. 2015; Cornelius et al. 2016). These field studies in the invaded regions in North America would test the pre-release predictions arising from the physiological and ecological host range studies conducted in the native range. These data will help to determine whether the introduction of $T$. japonicus into new areas invaded by $H$. halys is 'risky, or not'.

\section{Author contributions}

TH, PM, TG, and DG conceived research. JZ, FZ, and TH conducted the experiments. TH, TG, and PM wrote the manuscript. ET identified the egg parasitoids. All authors read and approved the manuscript.

Acknowledgements This work was funded by Agriculture and AgriFood Canada and China's donation to the CABI Development Fund. We would like to thank Marie-Claude Bon (USDA-ARS-EBCL, Campus International de Baillarguet, Montferrier le Lez, France) and Wenjun Bu (Nankai University, Tianjin, China) for helping with the identification of the parasitoids and stink bugs, respectively. We thank Hongyin Chen (Sino-American Biological Control Laboratory, Beijing, China) for providing Arma chinensis tested in our study. We also thank Chao Hou, Dandan Lu, Jian li, Yulin Yang, Ranran Jia, Kaijing $\mathrm{Hu}$, Haixia Zhan, Qianqian Mi, and Yongzhi Zhong for their assistance in implementing the field work.

\section{Compliance with ethical standards}

Conflict of interest The authors have declared that no conflict of interest exists.

Informed consent Informed consent was obtained from all individual participants included in the study.

Research involving human participants and/or animals This article does not contain any studies with human participants or animals (vertebrates) performed by any of the authors.

Open Access This article is distributed under the terms of the Creative Commons Attribution 4.0 International License (http://crea tivecommons.org/licenses/by/4.0/), which permits unrestricted use, distribution, and reproduction in any medium, provided you give appropriate credit to the original author(s) and the source, provide a link to the Creative Commons license, and indicate if changes were made.

\section{References}

Abram PK, Gariepy TD, Boivin G, Brodeur J (2014) An invasive stink bug as an evolutionary trap for an indigenous egg parasitoid. Biol Invasions 16:1387-1395

Arakawa R, Namura Y (2002) Effects of temperature on development of three Trissolcus spp. (Hymenoptera: Scelionidae), egg parasitoids of the brown marmorated stink bug, Halyomorpha halys (Hemiptera: Pentatomidae). Entomol Sci 5:215-218 
Bariselli M, Bugiani R, Maistrello L (2016) Distribution and damage by Halyomorpha halys in Italy. EPPO Bull 46:332-334

Barratt BIP, Evans AA, Ferguson CM, Barker GM, McNeill MR, Phillips CB (1997) Laboratory nontarget host range of the introduced parasitoids Microctonus aethiopoides and M. hyperodae (Hymenoptera: Braconidae) compared with field parasitism in New Zealand. Biol Control 26:694-702

Bergmann EJ, Venugopal PD, Martinson HM, Raupp MJ, Shrewsbury PM (2016) Host plant use by the invasive Halyomorpha halys (Stål) on woody ornamental trees and shrubs. PLoS ONE 11(2):e0149975

Bigler F, Babendreier D, Kuhlmann U (2006) Environmental impact of invertebrates for biological control of arthropods: methods and risk assessment. CABI Publishers, Wallingford

Colazza S, Lo Bue M, Lo Giudice D (2009) The response of Trissolcus basalis to footprint contact kairomones from Nezara viridula females is mediated by leaf epicuticular waxes. Naturwissenschaften 96:975-981

Cornelius ML, Dieckhoff C, Hoelmer KA, Olsen RT, Weber DC, Herlihy MV, Talamas EJ, Vineyard BT, Greenstone MH (2016) Biological control of sentinel egg masses of the exotic invasive stink bug Halyomorpha halys (Stål) in mid-Atlantic USA ornamental landscapes. Biol Contr 103:11-20

Corp. IBM (2013) IBM SPSS statistics for Windows, version 22.0. IBM Corp., Armonk

Duan JJ, Messing RH (1997) Biological control of fruit flies in Hawaii: factors affecting non-target risk analysis. Agric Hum Values 14:227-236

Follet PA, Duan J, Messing RH, Jones VP (2000) Parasitoid drift after biological control introductions: re-examining Pandora's box. Am Entomol 46:82-94

Froeschner RC (1988) Family Pentatomidae Leach, 1815. The stink bugs. In: Henry TJ, Froeschnern RC (eds) Catalog of the Heteroptera, or true bugs, of Canada and the Continental United States. E. J. Brill, Leiden, pp 544-607

Gariepy TD, Roitberg B (2013) New Tools in Biological Control. In: Mason PG, Gillespie DR (eds) Biological control programmes in Canada 2001-2012. CABI Publishing, Wallingford, UK, pp 22-37

Gariepy TD, Haye T, Zhang J (2013) Occurrence, genetic diversity, and potential pathways of entry of Halyomorpha halys in newlyinvaded areas of Canada and Switzerland. J Pest Sci 87:17-28

Gariepy TD, Haye T, Zhang J (2014) A molecular diagnostic tool for the preliminary assessment of host-parasitoid associations in biological control programs for a new invasive pest. Mol Ecol 23:3912-3924

Gariepy TD, Bruin A, Haye T, Milonas P, Vétek G (2015) Occurrence and genetic diversity of new populations of Halyomorpha halys in Europe. J Pest Sci 88:451-460

Haye T, Goulet H, Mason PG, Kuhlmann U (2005) Does fundamental host range match ecological host range?-A retrospective case study of a Lygus plant bug parasitoid. Biol Control 35:55-67

Haye T, Abdallah S, Gariepy T, Wyniger D (2014a) Phenology, life table analysis, and temperature requirements of the invasive brown marmorated stink bug, Halyomorpha halys, in Europe. J Pest Sci 87(3):407-418

Haye T, Wyniger D, Gariepy T (2014b) Recent range expansion of brown marmorated stink bug in Europe. In: Müller G, Pospischi R, Robinson WH (eds) In: Proceedings of the 8th international conference on urban pests, 20-23 July, Zurich, Switzerland, pp 309-314

Haye T, Fischer S, Zhang J, Gariepy TD (2015a) Can native egg parasitoids adopt the invasive brown marmorated stink bug, Halyomorpha halys (Heteroptera: Pentatomidae), in Europe? J Pest Sci 88:693-705
Haye T, Gariepy TD, Hoelmer K, Rossi JP, Streito JC, Tassus T, Desneux N (2015b) Range expansion of the invasive brown marmorated stinkbug, Halyomorpha halys: an increasing threat to field, fruit and vegetable crops worldwide. J Pest Sci $88: 665-673$

Hoebeke ER, Carter ME (2003) Halyomorpha halys (Stål) (Heteroptera: Pentatomidae): a polyphagous plant pest from Asia newly detected in North America. Proc Entomol Soc Wash 105:225-237

Howarth FG (1991) Environmental impacts of classical biological control. Ann Rev Entomol 36:485-509

Konopka JK, Haye T, Gariepy T, Mason P, Gillespie D, McNeil JN (2017) An exotic parasitoid provides an invasional lifeline for native parasitoids. Ecol Evol 7:277-284

Kuhlmann U, Schaffner U, Mason PG (2006) Selection of non-target species for host specificity tests. In: Bigler F, Babendreier D, Kuhlmann U (eds) Biological control of arthropods using invertebrates: methods for environmental risk assessment. CABI Publishers, Wallingford, pp 15-37

Lee DH, Short BD, Joseph SV, Bergh JC, Leskey TC (2013) Review of the biology, ecology, and management of Halyomorpha halys (Hemiptera: Pentatomidae) in China, Japan, and the Republic of Korea. Environ Entomol 42:627-641

Leskey TC, Hamilton GC, Nielsen AL et al (2012a) Pest status of the brown marmorated stink bug, Halyomorpha halys in the USA. Outlooks Pest Manag 23:218-226

Leskey TC, Lee DH, Short BD, Wright SE (2012b) Impact of insecticides on the invasive Halyomorpha halys (Hemiptera: Pentatomidae): analysis of insecticide lethality. J Econ Entomol 105:1726-1735

Martinson HM, Venugopal PD, Bergmann EJ, Shrewsbury PM, Raupp MJ (2015) Fruit availability influences the seasonal abundance of invasive stink bugs in ornamental tree nurseries. J Pest Sci 88:461-468

Mason PG, Kabaluk JT, Spencer B, Gillespie DR (2013) Regulation of biological control in Canada. In: Mason PG, Gillespie DR (eds) Biological control programmes in Canada 2001-2010. CABI Publishers, Wallingford, pp 1-5

Milnes JN, Wiman NG, Talamas EJ, Brunner JF, Hoelmer KA, Buffington ML, Beers EH (2016) Discovery of an exotic egg parasitoid of the brown marmorated stink bug Haylomorpha halys (Hemiptera: Pentatomindae) in the western United States. Proc Entomol Soc Wash 118:466-470

Qiu LF (2007) Studies on biology of the brown marmarated stink bug Halyomorpha halys (Stål) (Hemiptera:Pentatomidae), an important pest for pome trees in China and its biological control. Ph.D. dissertation, Chinese Academy of Forestry, Beijing

Qiu LF, Yang ZQ, Tao WQ (2007) Biology and population dynamics of Trissolcus halyomorphae. Sci Silvae Sin 43(11):62-65

Santacruz EN, Venette R, Dieckhoff C, Hoelmer K, Koch RL (2017) Cold tolerance of Trissolcus japonicus and T. cultratus, potential biological control agents of Halyomorpha halys, the brown marmorated stink bug. Biol Control 107:11-20

Talamas EJ, Buffington M, Hoelmer K (2013) New synonymy of Trissolcus halyomorphae Yang. J Hymenopt Res 33:113-117

Talamas EJ, Herlihy MV, Dieckhoff C, Hoelmer KA, Buffington ML, Bon M-C, Weber DC (2015) Trissolcus japonicus (Ashmead) emerges in North America. J Hymenopt Res 43:119-128

United States Apple Association (2010) Asian pest inflicting substantial losses, raising alarm in eastern apple orchards. Apple News 41:488

Van Driesche RG, Reardon R (2004) Assessing host ranges for parasitoids and predators used for classical biological control: a guide to best practice. Forest Health Technology Enterprise Team, USDA-Forest Service, Morgantown 
Van Lenteren JC, Bale J, Bigler F, Hokkanen HMT, Loomans AJM (2006) Assessing risks of releasing exotic biological control agents of arthropods pests. Ann Rev Entomol 51:609-634

Yang ZQ, Yao YX, Qiu LF, Li ZF (2009) A new species of Trissolcus (Hymenoptera: Scelionidae) parasitizing eggs of Halyomorpha halys (Heteroptera: Pentatomidae) in China with comments on its biology. Ann Entomol Soc Am 102:39-47

Zhu G, Bu W, Gao Y, Liu G (2012) Potential geographic distribution of brown marmorated stink bug invasion (Halyomorpha halys). PLoS ONE 7:e31246 